\title{
The prevalence of ESBL-producing Enterobacteriaceae in a nursing home setting compared with elderly living at home: a cross-sectional comparison
}

\author{
Andreas Blom, Jonas Ahl, Fredrik Månsson, Fredrik Resman and Johan Tham*
}

\begin{abstract}
Background: The aim of the study was to investigate the prevalence of faecal carriage of extended-spectrum beta-lactamase (ESBL)-producing Enterobacteriaceae among residents living in nursing homes and to compare it with a corresponding group of elderly people living in their own homes.

Methods: A total of 160 persons participated in the study between February and April 2014, 91 were residents in nursing homes $(n=10)$ and the remaining 69 were elderly living in their own homes. In addition to performing faecal samples, all participants answered a standardized questionnaire regarding known risk factors for ESBL-carriage.

Results: There was no significant difference between the groups, as 10 of the $91(11 \%)$ residents from nursing homes were ESBL-carriers compared with 6 of $69(8,7 \%)$ elderly living in their own homes. There was no significant difference between the groups. The total prevalence was $10 \%$. A univariate analysis revealed that the only studied risk factor significantly associated with ESBL-carriage was recent foreign travel $(p=0,017)$. All ESBL-positive isolates were Escherichia coli and there was a high degree of co-resistance to other antibiotics. All isolates $(n=17)$ were susceptible to imipenem and amikacin.

Conclusion: Residents of nursing homes as well as elderly living in their own homes have high rates of faecal carriage of ESBL-producing bacteria. These findings may affect the choice of empirical antibiotic treatment of severe infections in older adults.
\end{abstract}

Keywords: Elderly, ESBL, Nursing home, Enterobacteriaceae

\section{Background}

The rapid increase of ESBL-producing bacteria is a growing problem, and has been described as a pandemic [1]. Reported risk factors for carriage and infection include antibiotic treatment, age $\geq 65$ years, recent hospitalisation, prolonged hospital stay, recent surgery, recurrent UTIs, travel to foreign countries, severe illness, immobilization and residence at nursing home [2-8]. However, ESBLcarriage occurs in patients with no apparent risk factors as well [2, 9]. Age is a risk factor, but a Swedish study has shown that the median age among women infected with ESBL-producing bacteria has decreased from 62 to $52 \mathrm{y}$ in

\footnotetext{
* Correspondence: johan.tham@med.lu.se

Infectious Diseases Research Unit, Deptment of Clinical sciences, Lund University, Skånes University hospital, 20502 Malmö, Sweden
}

just five years, indicating a shifting epidemiological picture [10]. These risk factors are especially important to consider when treating patients with severe septicemia or septic shock empirically.

Even though the prevalence of ESBL-producing bacteria in Sweden is low in an international perspective, there has been a dramatic increase in the number of reported cases since 2007. In 2013 the number of reported cases were 8132, compared to the 7225 reported cases the previous year [11]. European Centre for Disease Prevention and Control (ECDC) registers and presents country prevalence data for resistant bacteria among invasive isolates, including Escherichia coli with resistance to third-generation cephalosporins from 30 European countries. The prevalence in Sweden was 
$4.4 \%$ in 2012, compared to some parts of southern Europe with a prevalence as high as $38.1 \%$ [12]. The prevalence of ESBL-carriage in the general population is not well-known, however. A Swedish study estimating the prevalence of ESBL-carriage among patients at Primary Health Care Units, which may represent a relatively healthy population, saw a rise from 2.1 to $3 \% 2010$, compared to various hospital wards where the prevalence increased from $1.8 \%$ in 2008 to $6.8 \%$ in 2010 [13]. Another Swedish study investigated the prevalence of ESBL-carriage among patients admitted to a surgery ward and found it to be $5 \%$ [14]. Studies of faecal carriage of ESBL-producing Enterobacteriaceae in nursing homes in different countries have showed a wide range of prevalence estimates, ranging from $3 \%$ (Sweden), $6.2 \%$ (Belgium), and $11.2 \%$ (Germany) to $40.5 \%$ in Northern Ireland [8, 15-17].

Infections with ESBL-producing Enterobacteriaceae have dire clinical and economical consequences, including higher mortality, higher costs and prolonged hospital stays [18-21]. The major risk associated with colonization is progress to infection among hospitalized patients [22]. This cohort also serves as a reservoir for ESBL-producing Enterobacteriaceae [22-24], increasing the risk of spread to other patients, especially when there is poor adherence to hygiene routines $[22,23,25,26]$. A Swedish study analyzed the ESBL-producing Enterobacteriaceae in a nursing home setting via pulsed-field gel electrophoresis (PFGE) and the result indicated a person-to-person transmission via the staff's hands or clothes [15]. A further risk for patients infected with ESBL-producing Enterobacteriaceae is receiving inadequate empirical antibiotic treatment [27], which is the main reason behind the higher mortality in this group.

Only elderly that are requiring significantly care $24 / 7$ are staying at nursing homes in Sweden since we have a well-developed tradition to take care of elderly at their own homes as long as possible. At nursing homes in Sweden there are usually nurses during daytime and doctors linked to the homes. The aim of this study was to investigate the prevalence of fecal ESBL-carriage in a nursing home setting and compare it with a corresponding population of elderly that still live in their own homes.

\section{Methods}

\section{Study design}

The study was designed as a cross-sectional comparison study in February to April 2014 to investigate the prevalence of fecal carriage of ESBL-producing Enterobacteriaceae in a nursing home setting compared with elderly living in their own homes. The study was carried out in the cities of Malmö and Lund in Sweden. In Malmö and Lund there are 59 and 16 nursing homes, respectively. Nursing homes that exclusively housed residents with dementia were excluded. A total of 30 nursing homes were contacted. In each of these nursing homes, a contact person (usually a nurse) provided a list of potential participants with the ability to provide informed consent. Elderly that had stayed at the nursing home of one month or more were eligible for inclusion. Each resident had a room of his or her own with separate toilet and shower.

Elderly with an age $>60$ y living in their own homes were approached on a total of 8 meeting places for elderly. Ethical approval for the study was obtained from the Research Ethics Committee of the University of Lund 2013/898.

\section{Data collection}

Rectal bacterial samples were acquired using a rectal swab pin (eSwab, Copan, Brescia, Italy). In the nursing homes, the samples were collected by a medical student. In the meeting places for the elderly the participants were collecting the samples themselves after a standardized instruction. One sample per person was obtained.

Every participant answered a standardized questionnaire regarding foreign travel, treatment with antibiotics, surgical procedures or hospital stay the last six months and prevalence of chronic heart disease, COPD, other chronic lung disease, neurological disease and stroke, diabetes mellitus, autoimmune disease, active cancer, hematological disease, liver failure, kidney failure, immunosuppressive treatment corresponding a daily dose of $\geq 5 \mathrm{mg}$ prednisolone and length of stay at the nursing home. When needed, this information was obtained from the nurse in charge.

\section{Microbiological methods}

All samples were transported in Amies medium (Copan, Brescia, Italy) to the Laboratory of Microbiology at Malmö University Hospital within $24 \mathrm{~h}$. The samples were inoculated on agar plates with medium selective for cephalosporin resistance (ChromID ESBL, Bio-Mérieux $\mathrm{x}^{\mathrm{Tm}}$ ). All isolates obtained from these plates were further examined for ESBL production through synergy testing on MüllerHinton agar plate (Oxoid Thermo Fischer) with discs containing cefpodoxime and ESBL- and AmpC-inhibitor (AmpC \& ESBL Detection Set, Mast Group Ltd, Meyerside, UK). Antimicrobial susceptibility testing was performed on the ESBL-positive isolates using the disk diffusion method according to the Swedish Reference Group of Antibiotics method $[28,29]$. The species determination was performed using MALDI-TOF. MIC determination was performed using E-test strips (Biomerieux, Marcy-l'Étoile, France).

\section{Statistics}

Statistical analysis was performed using IBM SPSS version 22 (IBM Corporation, New York, USA). The analysis of risk factors was performed using Pearson's $X^{2}$ or Fischer's exact test, and $95 \%$ confidence intervals were 
calculated using the method of Clopper and Pearson. Man$\mathrm{n}$-Whitney $U$ test was performed calculating $\mathrm{p}$-values in age between both groups. An unadjusted $p$-value $<0.05$ was considered statistically significant.

\section{Results}

A total of 30 nursing homes were approached, 18 in Malmö and 12 in Lund. Of these, ten decided to participate, seven in Malmö and three in Lund. A total of 91 residents were recruited and subsequently screened. Ten residents denied participation after the information about the study was given. In the group of elderly living at home a total of 69 individuals were screened after they accepted participation. A total of 160 people were screened.

\section{Descriptive data}

The median age in the nursing home cohort and control cohort was 89 and 78 years, respectively. The median age of the two groups together was 85 years. There was a female dominance in both groups, 69 and $74 \%$ respectively. The descriptive characteristics and the results from the questionnaire in the two cohorts is shown in Table 1 In the nursing home cohort, the univariate analysis revealed significantly more hospital stays and recent antibiotic treatments, as well as higher proportions of chronic heart disease, COPD and neurological diseases. There was a difference in age where occupants at nursing homes were significantly older than study participants living in their own homes $(p<0.0001)$. No age differences were seen between ESBL and non-ESBL carriers for the whole study population, nor within the nursing-home or the non-nursing home group. However, international travel was significantly more frequent among the elderly living in their own homes $(p=<0.001$, Table 1$)$.

\section{Prevalence of ESBL-carriage}

The total prevalence of faecal carriage of ESBL-producing Enterobacteriaceae was 10 \% (16/160; 95 \% CI 5.8-15.7 \%). In the nursing home cohort ten residents were ESBLcarriers, i.e. $11 \%$ (95 \% CI 5.9-19.2\%), and in the control cohort six individuals tested positive (8.7 \% (95 \% CI 3.718 The median age for the ESBL-carriers in the respective cohorts were 90 and 70.5 years. The majority of the carriers of ESBL-positive bacteria were women, $69 \%$, which is representative for the two groups (Table 2). The difference in prevalence between the two groups is not statistically significant $(p=0.632)$. In four of the ten nursing homes ESBLproducing bacteria were not isolated at all.

\section{Information on isolated bacteria}

All of the isolates of the ESBL-producing Enterobacteriaceae were E. coli. One person was carrying two different isolates with different resistance pattern. All of the ESBL- producing isolates $(n=17)$ were susceptible to imipenem and amikacin. The resistance pattern of the strains is shown in Table 3. Nine out of the 17 isolates were resistant against $\geq 2$ antibiotic classes in addition to betalactam antibiotics.

\section{Risk factors for carriage of ESBL-producing E. Coli}

Six out of 16 ESBL-carriers had travelled abroad in the past 6 months and five of these individuals were elderly living in their own homes. International travel was the only statistically significant association with ESBLcarriage $(p=0.017)$. Six persons had received antibiotic treatment in the past 6 months, all of them residents in nursing homes. For one of the ESBL-carriers, no apparent risk factor for ESBL-carriage was identified.

\section{Discussion}

In this cross-sectional comparison study we found no difference in the prevalence of ESBL-producing Enterobacteriaceae among nursing home residents and the elderly living in their own homes (11 and $8.7 \%$ respectively). We found that a total of $10 \%$ were ESBL-carriers among all of the elderly in the study, which was higher than expected.

Our investigation has limitations. We did not perform PFGE, rep-PCR or MLST on isolates to examine the possibility of spread of the ESBL-producing Enterobacteriaceae between the elderly. However, an analysis of the respective resistance patterns was performed, and it showed widespread resistance to trimethoprim-sulfametoxazole and ciprofloxacin, two widely used antibiotics against common infections like UTIs. Another limitation is that only faecal sample per participant was collected, and an intermittent secretion of ESBL-producing bacteria could theoretically have been missed [17]. In total 160 persons were screened, a higher number would have increased the statistical power and perhaps the results could have been different. We don't think so due to the fact that the healthy elderly is travelling a lot (in Sweden) and this seems to be of just as high importance to become rectal carrier of ESBL: $s$ as staying at a nursing home. However, the risk for a type II error cannot be fully excluded in this material.

The two major reasons for not participate in the study was due to shortage of staff and a high ratio of residents suffering from dementia. The shortage of staff was an argument from some of the nursing homes that declined to participate, but we haven't performed a survey over the staffing levels in the actual nursing homes. The size of the nursing home were not an important factor regarding participation in the study. Rooney et al. have hypothesized that nursing homes that participate in this kind of study are the ones that are sufficiently staffed and therefore are better equipped to observe hygiene routines [17]. In line with this, Andersson et al. reports that lack of time is the main reason why infection 
Table 1 Demographics and risk factors

\begin{tabular}{|c|c|c|c|}
\hline Variable & Nursing home $(n=91)$ & Living at home $(n=69)$ & $P$-value \\
\hline Age (years) & $65-102$ & $60-98$ & - \\
\hline Age median (years) & 89 & 78 & - \\
\hline Female & $63(69 \%)$ & $51(74 \%)$ & - \\
\hline Male & $28(31 \%)$ & $18(26 \%)$ & - \\
\hline Hospital stay ${ }^{a}$ & $15(16.5 \%)$ & $3(4.3 \%)$ & 0,016 \\
\hline Surgery $^{a}$ & $2(2.2 \%)$ & $2(2.9 \%)$ & 1 \\
\hline Foreign travel ${ }^{a}$ & $1(1.1 \%)$ & $23(33.3 \%)$ & $<0,001$ \\
\hline Hospital stay abroad ${ }^{a}$ & 0 & 0 & - \\
\hline Chronic heart disease & $31(34.1 \%)$ & $11(15.9 \%)$ & 0,01 \\
\hline COPD & $10(11.0 \%)$ & $1(1.4 \%)$ & 0,024 \\
\hline Other chronic lung disease & $5(5.5 \%)$ & $4(5.8 \%)$ & 1.00 \\
\hline Neurological disease, stroke & $23(25.3 \%)$ & $6(8.7 \%)$ & 0.007 \\
\hline Diabetes mellitus & $7(7.7 \%)$ & $10(14.5 \%)$ & 0.167 \\
\hline Autoimmune disease & $8(8.8 \%)$ & $3(4.3 \%)$ & 0.353 \\
\hline Cancer & $10(11.0 \%)$ & $2(2.9 \%)$ & 0.054 \\
\hline Haematological disease & $1(1.1 \%)$ & 0 & 1.00 \\
\hline Liver failure & $1(1.1 \%)$ & 0 & 1.00 \\
\hline Kidney failure & $3(3.3 \%)$ & 0 & 0.26 \\
\hline Immunosuppressive treatment ${ }^{b}$ & 0 & 0 & - \\
\hline Antibiotic treatment ${ }^{a}$ & $25(27.5 \%)$ & $5(7.2 \%)$ & 0.001 \\
\hline
\end{tabular}

${ }^{\mathrm{a}}$ The past six months ${ }^{\mathrm{b}}$ Corresponding Prednisolon $>5 \mathrm{mg} /$ day

preventing routines are not followed [15], which increases the risk of spreading potential ESBL-producing bacteria via the staff's hands or clothes $[9,15]$. Only 91 of 434 residents in the nursing homes were screened. The main reasons for this loss was dementia diagnosis, but also severe illness and immobilization of the residents. Immobility, comorbidity and severe illness are risk factors for colonization with ESBL-producing Enterobacteriaceae, and not being able to include the residents with these risk factors might affect the result $[4,8]$. In the group of elderly staying at home, when we finally found them there were only a few who declined participation so we did not perform any non-response analyzation.

Many nursing home residents per definition have risk factors for ESBL colonization and living at a nursing home is a risk factor per se $[2,4]$. In this study, the ESBL-positive residents at nursing homes had between

Table 2 Characteristics ESBL-positive $(n=16)$

\begin{tabular}{llll}
\hline Participants & Nursing home & Living at home & Total \\
\hline Female $n(\%)$ & $7(70)$ & $4(67)$ & $11(69)$ \\
Male $n(\%)$ & $3(30)$ & $2(33)$ & $5(31)$ \\
Total $n(\%)$ & $10(63)$ & $6(37)$ & $16(100)$ \\
Age (years) & $75-97$ & $67-81$ & $67-97$ \\
Age (years) median & 90 & 70,5 & 83,5 \\
\hline
\end{tabular}

two and four established risk factors for colonization. Assessment of risk factors is therefore an important instrument to find patients who could be at risk of being colonized with ESBL-producing Enterobacteriaceae.

Nine of the 17 ESBL-positive isolates were resistant to $\geq 2$ antibiotic classes in addition to betalactam antibiotics, which may complicate the treatment of common infections. The most common findings were resistance to ciprofloxacin and trimethoprim-sulfametoxazole, both antibiotics commonly used against UTIs and one has to consider the choice of antibiotics against this condition to get full effect and not to drive the resistance.

There was a female dominance in both study populations, and this corresponds partly with the representation of gender in Sweden among the elderly: in 2013, $60 \%$ of individuals in the ages between 80 and 89 years [30] were female. The difference in age between the ESBL-positive in the two groups were 20 years (median age 90 and 70.5 years respectively), which in part reflects the difference in age between the two populations, but it also suggests the co-existence of different categories of individuals that become colonized with ESBL-producing Enterobacteriaceae, with different risk factors. Brolund et al. has shown that the median age of infected female patients in Sweden has decreased from 62 to 52 y between 2007 and 2011, which indicates a higher number of younger, healthier people being colonized with ESBL- 
Table 3 Antibiotic resistance ESBL-producing E. coli-isolates

\begin{tabular}{ll}
$(n=17)$ & $\begin{array}{l}\text { Resistance in ESBL-producing } \\
\text { E.coli-isolates }(n=17)\end{array}$ \\
\hline Antibiotic & $24 \%$ \\
\hline Gentamicin & $35 \%$ \\
Tobramycin & $0 \%$ \\
Amikacin & $0 \%$ \\
Imipenem & $77 \%$ \\
Trimethoprim-sulfametoxazole & $59 \%$ \\
Ciprofloxacin & $6 \%$ \\
\hline Piperacillin-Tazobactam &
\end{tabular}

producing Enterobacteriaceae through communityacquisition, which is a shift from earlier clinical picture where the nosocomial setting with elderly patients with comorbidity dominated [10]. Five of the six elderly living at home that were ESBL-positive had foreign travel as risk factor, whereof four had travelled outside the Nordic countries. Foreign travel was the only statistically significant connection to ESBL-carriage $(p=0.017)$, and has in Sweden been reported to be a major risk factor for ESBL acquisition, [24].

To our knowledge there is only one earlier study of the prevalence of faecal carriage of ESBL-producing Enterobacteriaceae in a nursing home setting in Sweden 2008 , and this showed a prevalence of $3 \%$ [15]. Other European studies have shown prevalence numbers ranging from 6.2 to $40.5 \%[8,17]$. These numbers, along with our result, indicates that nursing homes may act as a reservoir for ESBL-producing bacteria. These results should be taken in consideration when treating these groups of patients if they develop severe sepsis or septic shock.

\section{Conclusions}

The prevalence of ESBL-producing Enterobacteriaceae in the nursing homes studied has increased, but not more than among the healthy elderly population in general. This depends mainly on the fact that there are different risk factors in these two groups, and foreign travel-an established risk factor-has become far more common among healthy elderly people, leading to an increased risk of being colonized with ESBL-producing Enterobacteriaceae when travelling to countries and areas where the prevalence is higher. The risk of infection caused by ESBL-producing Enterobacteriaceae also increases the risk of getting inadequate initial empirical antibiotic treatment, which in turn increases the mortality in severe infections $[18,20,21]$. Our study showed that 1 of 10 elderly people carry ESBL-producing $E$. coli, which questions the current empiric antibiotic treatment of elderly with certain risk factors [27]. It would be desirable in future studies to include the residents with dementia or immobilization, to have a fuller picture, and to have a better match between the two groups regarding age and risk factors to be able to help these vulnerable individuals in the future.

\section{Abbreviations}

COPD: chronic obstructive pulmonary disease; ECDC: European Centre for Disease Prevention and Control; ESBL: extended-spectrum beta-lactamase; PFGE: pulsed-field gel electrophoresis; UTI: urinary tract infection.

\section{Competing interests}

We declare no competing interests for this study.

\section{Authors' contributions}

AB collected most of the data. FM, FR, JA and JT designed the study. All helped to interpret and analyze the data. All also participated in the drafting and revision of the manuscript and added substantive intellectual contributions. All authors read and approved the final manuscript.

\section{Acknowledgements}

We wish to acknowledge the assistance of the following individuals: Carina Linder, Lund University and SUS Skåne and all of the nurses at the different nursing homes in Malmö for helping us with the study.

Received: 3 June 2015 Accepted: 11 February 2016

Published online: 04 March 2016

References

1. Pitout JD, Laupland KB. Extended-spectrum beta-lactamase-producing enterobacteriaceae: an emerging public-health concern. Lancet Infect Dis. 2008;8(3):159-66.

2. Ben-Ami R, Rodriguez-Bano J, Arslan H, Pitout JD, Quentin C, Calbo ES, et al. A multinational survey of risk factors for infection with extended-spectrum beta-lactamase-producing enterobacteriaceae in nonhospitalized patients. Clin infect dis : an official publication of the Infectious Diseases Society of America. 2009;49(5):682-90.

3. Colodner R, Rock W, Chazan B, Keller N, Guy N, Sakran W, et al. Risk factors for the development of extended-spectrum beta-lactamase-producing bacteria in nonhospitalized patients. Eur j clin microbiolinfectdis : official publication of the European Society of Clinical Microbiology. 2004;23(3): 163-7.

4. Pitout JD. Infections with extended-spectrum beta-lactamase-producing enterobacteriaceae: changing epidemiology and drug treatment choices. Drugs. 2010;70(3):313-33.

5. Tangden T, Cars O, Melhus A, Lowdin E. Foreign travel is a major risk factor for colonization with escherichia coli producing CTX-M-type extendedspectrum beta-lactamases: a prospective study with swedish volunteers. Antimicrob Agents Chemother. 2010;54(9):3564-8.

6. Tham J, Odenholt I, Walder M, Andersson L, Melander E. Risk factors for infections with extended-spectrum beta-lactamase-producing escherichia coli in a county of Southern Sweden. Infection and drug resistance. 2013;6:93-7.

7. Tham J, Odenholt I, Walder M, Brolund A, Ahl J, Melander E. Extendedspectrum beta-lactamase-producing escherichia coli in patients with travellers' diarrhoea. Scand J Infect Dis. 2010;42(4):275-80.

8. Gruber I, Heudorf U, Werner G, Pfeifer Y, Imirzalioglu C, Ackermann H, et al. Multidrug-resistant bacteria in geriatric clinics, nursing homes, and ambulant care_-prevalence and risk factors. Intj med microbiol: IJMM. 2013; 303(8):405-9.

9. Stuart RL, Kotsanas D, Webb B, Vandergraaf S, Gillespie EE, Hogg GG, et al. Prevalence of antimicrobial-resistant organisms in residential aged care facilities. Med J Aust. 2011;195(9):530-3.

10. Brolund A, Edquist PJ, Makitalo B, Olsson-Liljequist B, Soderblom T, Wisell KT, et al. Epidemiology of extended-spectrum beta-lactamase-producing Escherichia coli in Sweden 2007-2011. Clinical microbiology and infection: the official publication of the European Society of Clinical Microbiology and Infectious Diseases. 2014;20(6):0344-52.

11. Hellman J O-LB, Bengtsson B, Greko C. SWEDRES/SVARM 20132013 [12 November 2014]. Available from: http://www.folkhalsomyndigheten.se/ pagefiles/17612/Swedres-Svarm-2013.pdf. Accessed: 25/11/ 2014. 
12. European Centre for Disease Prevention and Control [28 April 2014]. Available from: http://www.ecdc.europa.eu/en/healthtopics/antimicrobial_ resistance/database/Pages/database.aspx. Accessed: 25/11/ 2014.

13. Stromdahl $H$, Tham J, Melander E, Walder M, Edquist PJ, Odenholt I. Prevalence of faecal ESBL carriage in the community and in a hospital setting in a county of Southern Sweden. Eur $\mathrm{j}$ clin microbiol infect dis : official publication of the European Society of Clinical Microbiology. 2011; 30(10):1159-62.

14. Chabok A, Tarnberg M, Smedh K, Pahlman L, Nilsson LE, Lindberg C, et al. Prevalence of fecal carriage of antibiotic-resistant bacteria in patients with acute surgical abdominal infections. Scand J Gastroenterol. 2010;45(10): 1203-10.

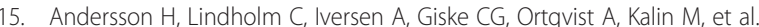
Prevalence of antibiotic-resistant bacteria in residents of nursing homes in a swedish municipality: healthcare staff knowledge of and adherence to principles of basic infection prevention. Scand J Infect Dis. 2012;44(9):641-9.

16. Jans B, Schoevaerdts $D$, Huang TD, Berhin C, Latour K, Bogaerts $P$, et al. Epidemiology of multidrug-resistant microorganisms among nursing home residents in Belgium. PLoS One. 2013;8(5), e64908.

17. Rooney PJ, O'Leary MC, Loughrey AC, McCalmont M, Smyth B, Donaghy P, et al. Nursing homes as a reservoir of extended-spectrum beta-lactamase (ESBL)-producing ciprofloxacin-resistant escherichia coli. J Antimicrob Chemother. 2009;64(3):635-41.

18. Giske CG, Monnet DL, Cars O, Carmeli Y. Clinical and economic impact of common multidrug-resistant gram-negative bacilli. Antimicrob Agents Chemother. 2008:52(3):813-21.

19. Lautenbach E, Patel JB, Bilker WB, Edelstein PH, Fishman NO. Extendedspectrum beta-lactamase-producing escherichia coli and klebsiella pneumoniae: risk factors for infection and impact of resistance on outcomes. Clin infect dis : an official publication of the Infectious Diseases Society of America. 2001;32(8):1162-71.

20. Melzer M, Petersen I. Mortality following bacteraemic infection caused by extended spectrum beta-lactamase (ESBL) producing E. Coli compared to non-ESBL producing E. Coli. J Infect. 2007;55(3):254-9.

21. Schwaber MJ, Carmeli Y. Mortality and delay in effective therapy associated with extended-spectrum beta-lactamase production in enterobacteriaceae bacteraemia: a systematic review and meta-analysis. J Antimicrob Chemother. 2007;60(5):913-20.

22. Valverde A, Grill F, Coque TM, Pintado V, Baquero F, Canton R, et al. High rate of intestinal colonization with extended-spectrum-beta-lactamaseproducing organisms in household contacts of infected community patients. J Clin Microbiol. 2008;46(8):2796-9.

23. Oteo J, Perez-Vazquez M, Campos J. Extended-spectrum [beta]-lactamase producing escherichia coli: changing epidemiology and clinical impact. Curr Opin Infect Dis. 2010;23(4):320-6.

24. Woerther PL, Burdet C, Chachaty E, Andremont A. Trends in human fecal carriage of extended-spectrum beta-lactamases in the community: toward the globalization of CTX-M. Clin Microbiol Rev. 2013;26(4):744-58.

25. Hilty M, Betsch BY, Bogli-Stuber K, Heiniger N, Stadler M, Kuffer M, et al. Transmission dynamics of extended-spectrum beta-lactamase-producing enterobacteriaceae in the tertiary care hospital and the household setting. Clin infect dis : an official publication of the Infectious Diseases Society of America. 2012;55(7):967-75.

26. Rodriguez-Bano J, Lopez-Cerero L, Navarro MD, Diaz de Alba P, Pascual A. Faecal carriage of extended-spectrum beta-lactamase-producing escherichia coli: prevalence, risk factors and molecular epidemiology. J Antimicrob Chemother. 2008:62(5):1142-9.

27. Van Aken S, Lund N, Ahl J, Odenholt I, Tham J. Risk factors, outcome and impact of empirical antimicrobial treatment in extended-spectrum beta-lactamase-producing escherichia coli bacteraemia. Scand J Infect Dis. 2014:46(11):753-62.

28. Referensgruppen för antibiotikafrågor [19 maj 2014]. Available from: http:// referensmetodik.folkhalsomyndigheten.se/w/Huvudsida. Accessed: 25/11/ 2014

29. Donaldson H, McCalmont M, Livermore DM, Rooney PJ, Ong G, McHenry E, et al. Evaluation of the VITEK 2 AST N-054 test card for the detection of extended-spectrum beta-lactamase production in escherichia coli with CTX-M phenotypes. J Antimicrob Chemother. 2008;62(5):1015-7.

30. Statistiska Centralbyrån 2014 [22 maj 2014]. Available from: http://www. statistikdatabasen.scb.se. Accessed: 25/11/ 2014.

\section{Submit your next manuscript to BioMed Central and we will help you at every step:}

- We accept pre-submission inquiries

- Our selector tool helps you to find the most relevant journal

- We provide round the clock customer support

- Convenient online submission

- Thorough peer review

- Inclusion in PubMed and all major indexing services

- Maximum visibility for your research

Submit your manuscript at www.biomedcentral.com/submit
Biomed Central 